JouRnal OF Universal Mathematics

Vol.1 No.2 PP.204-215 (2018)

ISSN-2618-5660

\title{
MULTIPLICATION OPERATION AND POWERS OF TRAPEZOIDAL FUZZY NUMBERS
}

\author{
E. ELJAOUI, S. MELLIANI, AND L. S. CHADLI
}

\begin{abstract}
In this work, we explore and exhibe some numerical methods to calculate two types of the product (respectively powers) of trapezoidal fuzzy numbers. As a particular case, we study the multiplication operation on triangular fuzzy numbers, we report and correct errors in an article dealing with the same topic.
\end{abstract}

\section{INTRODUCTION}

The fuzzy numbers theory is used in different domains, in linguistics, statistics, engineering, physics, biology and experimental sciences. In 1965, Zadeh introduced the fuzzy sets theory. Then, many works was devoted to the study of arithmetic operations on fuzzy numbers. Especially, Dubois and Prade [2] defined a fuzzy number as a subset of the real line [4].

In [1], Bansal studied the arithmetic behavior of trapezoidal fuzzy numbers and formulated the basic mathematical operations on this type of fuzzy numbers.

Gao et al. [4] used the extension principle to develop nonlinear programming method, analytical method, computer drawing method and computer simulation method for solving multiplication operation of two fuzzy numbers.

In [9], Mahanta et al. introduced an alternative method to evaluate the arithmetic operations on triangular fuzzy numbers.

In this paper, we report and correct some errors in the article [4], and we develop numerical methods to calculate the inner and canonical products of trapezoidal and triangular fuzzy numbers.

In section 2 , we give some preliminaries on fuzzy numbers and trapezoidal fuzzy numbers and we correct an error in [4]. In section 3, we apply analytical method, computer drawing method and computer simulation method for solving multiplication operation of two trapezoidal fuzzy numbers. In section 4, we study the square and the nth power of trapezoidal fuzzy numbers using inner and canonical multiplication.

\section{Preliminaries}

A fuzzy number is a fuzzy set on the real axis, i.e. a mapping $u: \mathbb{R} \longrightarrow[0,1]$ which satisfies the following four conditions:

Date: June 1, 2018, accepted. 
(i) $u$ is normal, i.e. $\exists x_{0} \in \mathbb{R}$ for which $u\left(x_{0}\right)=1$,

(ii) $u$ is fuzzy convex, i.e. $u(\lambda x+(1-\lambda) y) \geq \min (u(x), u(y))$ for any $x, y \in$ $\mathbb{R}$ and $\lambda \in[0,1]$

(iii) $u$ is upper semi-continuous,

(iv) The closure $[u]^{0}=\overline{\{x \in \mathbb{R} \mid u(x)>0\}}$ of the support supp $u=\{x \in \mathbb{R} \mid u(x)>0\}$ of $u$ is compact.

We denote the set of all fuzzy numbers on $\mathbb{R}$ by $E$ and called it as the space of fuzzy numbers. For $0<\alpha \leq 1$, denote $[u]_{\alpha}=\{x \in \mathbb{R} \mid u(x) \geq \alpha\}$.

Then, from (i)-(iv), it follows that the $\alpha$-level set $[u]_{\alpha}$ is a compact interval $[u]_{\alpha}=$ $\left[u^{-}(\alpha), u^{+}(\alpha)\right]$, for all $0 \leq \alpha \leq 1$.

It is well known that the following properties are true

$$
[u+v]_{\alpha}=[u]_{\alpha}+[v]_{\alpha}, \quad[k u]_{\alpha}=k[u]_{\alpha},
$$

and

$$
[u v]_{\alpha}=[u]_{\alpha}[v]_{\alpha},
$$

for each $u, v \in E, k \in \mathbb{R}$ and $\alpha \in[0,1]$.

The following theorem known as "Representation Theorem" is very important.

Theorem 2.1. Let $[u]_{\alpha}=\left[u^{-}(\alpha), u^{+}(\alpha)\right]$, for $u \in E$ and for all $0 \leq \alpha \leq 1$. Then the following statements hold:

(i) $u^{-}(\alpha)$ is a bounded non-decreasing left continuous function in $(0,1]$.

(ii) $u^{+}(\alpha)$ is a bounded non-increasing left continuous function in $(0,1]$.

(iii) $u^{-}(\alpha)$ and $u^{+}(\alpha)$ are right continuous at $\alpha=0$.

(iv) $u^{-}(1) \leq u^{+}(1)$.

A crisp number $k$ is simply represented by $u^{-}(\alpha)=u^{+}(\alpha)=k$, for all $0 \leq \alpha \leq 1$. A triangular fuzzy number is represented by three reals as follows $\widetilde{A}=\left(a_{1}, a_{2}, a_{3}\right)$ and its membership function $\mu_{\widetilde{A}}$ is given by

$$
\mu_{\widetilde{A}}(x)=\left\{\begin{array}{ccc}
0 & \text { si } & x<a_{1} \\
\frac{x-a_{1}}{a_{2}-a_{1}} & \text { si } & a_{1} \leq x \leq a_{2} \\
\frac{a_{3}-x}{a_{3}-a_{2}} & \text { si } & a_{2} \leq x \leq a_{3} \\
0 & \text { si } & x>a_{3}
\end{array}\right.
$$

Please notice that the membership function of a triangular fuzzy number is piecewise affine.

The $\alpha$-cut of a triangular fuzzy number $\widetilde{A}=\left(a_{1}, a_{2}, a_{3}\right)$ is given by $A_{\alpha}=\left[a_{L}^{(\alpha)}, a_{R}^{(\alpha)}\right]=$ $\left[a_{1}+\left(a_{2}-a_{1}\right) \alpha, a_{3}-\left(a_{3}-a_{2}\right) \alpha\right]$, with $\alpha \in[0,1]$.

Definition 2.2. A trapezoidal fuzzy number is represented by four real numbers as follows $\widetilde{A}=(a, b, c, d)$, with $a \leq b \leq c \leq d$ and its membership function $\mu_{\widetilde{A}}$ is given by

$$
\mu_{\widetilde{A}}(x)=\left\{\begin{array}{ccc}
\frac{x-a}{b-a} & \text { if } & a \leq x \leq b \\
1 & \text { if } & b \leq x \leq c \\
\frac{d-x}{d-c} & \text { si } & c \leq x \leq d \\
0 & & \text {, otherwise }
\end{array}\right.
$$

This fuzzy number is said to be positive (respectively negative) if $a \geq 0$ (respectively $d \leq 0$ ). 
A trapezoidal fuzzy number $\widetilde{A}=(a, b, c, d)$ such that $b=c$ is a triangular fuzzy number.

\section{Definition 2.3. Operations on trapezoidal fuzzy numbers}

Let $\widetilde{A}=(a, b, c, d)$ and $\widetilde{B}=(e, f, g, h)$ be two trapezoidal fuzzy numbers, we define

(i) $\widetilde{A} \oplus \widetilde{B}=(a+e, b+f, c+g, d+h)$.

(ii) $\widetilde{A} \ominus \widetilde{B}=(a-h, b-g, c-f, d-e)$.

(iii) $-\widetilde{A}=(-d,-c,-b,-a)$.

We define two type of multiplication for trapezoidal fuzzy numbers:

- the first type is based on Zadeh's extension principle, which we will denote by $\times$,

- and the second one is defined in a way to results in a trapezoidal fuzzy number, which we will denote by $\otimes$.

\section{Definition 2.4. Canonical multiplication}

Let $\widetilde{A}=(a, b, c, d)$ and $\widetilde{B}=(e, f, g, h)$ be two trapezoidal fuzzy numbers. Using Zadeh's extension principle, we define their canonical product $\widetilde{Q}=\widetilde{A} \times \widetilde{B}$ by its $\alpha$-cuts

\section{Definition 2.5. Inner multiplication}

$$
\widetilde{Q}_{\alpha}=\widetilde{A}_{\alpha} \times \widetilde{B}_{\alpha}
$$

Let $\widetilde{A}=(a, b, c, d)$ and $\widetilde{B}=(e, f, g, h)$ be two trapezoidal fuzzy numbers, we define their inner product $\widetilde{A} \otimes \widetilde{B}$ by

$\widetilde{A} \otimes \widetilde{B}=(\min \{a e, a h, d e, d h\}, \min \{b f, c f, c g, b g\}, \max \{b f, c f, c g, b g\}, \max \{a e, a h, d e, d h\})$.

\section{Multiplication Operation on Trapezoidal Fuzzy Numbers}

The procedure of addition or substraction is simple, but the procedure of multiplication or division is difficult and complex.

Now we extend some numerical methods presented in [4] for solving multiplication operation of two triangular fuzzy numbers, which we apply for the multiplication of two trapezoidal fuzzy numbers.

\subsection{Analytical Method.}

\subsubsection{Canonical multiplication.}

Let $\widetilde{A}=(a, b, c, d), \widetilde{B}=(e, f, g, h)$ be two trapezoidal fuzzy numbers and $\widetilde{Q}=\widetilde{A} \times \widetilde{B}$. We have $\widetilde{A}_{\alpha}=\left[a_{\alpha}^{L}, a_{\alpha}^{R}\right]=[a+\alpha(b-a), d+\alpha(c-d)]$ and $\widetilde{B}_{\alpha}=\left[b_{\alpha}^{L}, b_{\alpha}^{R}\right]=$ $[e+\alpha(f-e), h+\alpha(g-h)]$. Then, $\widetilde{Q}_{\alpha}=\left[a_{\alpha}^{L}, a_{\alpha}^{R}\right] \times\left[b_{\alpha}^{L}, b_{\alpha}^{R}\right]=\left[q_{\alpha}^{L}, q_{\alpha}^{R}\right]$, where $q_{\alpha}^{L}=$ $\min \left\{a_{\alpha}^{L} b_{\alpha}^{L}, a_{\alpha}^{L} b_{\alpha}^{R}, a_{\alpha}^{R} b_{\alpha}^{L}, a_{\alpha}^{R} b_{\alpha}^{R}\right\}$ and $q_{\alpha}^{R}=\max \left\{a_{\alpha}^{L} b_{\alpha}^{L}, a_{\alpha}^{L} b_{\alpha}^{R}, a_{\alpha}^{R} b_{\alpha}^{L}, a_{\alpha}^{R} b_{\alpha}^{R}\right\}$. We suppose that the trapezoidal fuzzy numbers $\widetilde{A}$ and $\widetilde{B}$ are positive, then $a_{\alpha}^{L}, a_{\alpha}^{R}, b_{\alpha}^{L}, b_{\alpha}^{R}$ are positive. Therefore

$$
\begin{aligned}
q_{\alpha}^{L} & =a_{\alpha}^{L} b_{\alpha}^{L} \\
& =(a+\alpha(b-a))(e+\alpha(f-e)) \\
q_{\alpha}^{L} & =(b-a)(f-e) \alpha^{2}+(a f+b e-2 a e) \alpha+a e
\end{aligned}
$$

substituting $q_{\alpha}^{L}=z$, we get

$$
\alpha=\frac{-(a f+b e-2 a e)+\sqrt{(a f-b e)^{2}+4(b-a)(f-e) z}}{2(b-a)(f-e)}, \quad(\text { Omit } \alpha<0) .
$$


Similarly, we have

$$
\begin{aligned}
q_{\alpha}^{R} & =a_{\alpha}^{R} b_{\alpha}^{R} \\
& =(d+\alpha(c-d))(h+\alpha(g-h)) \\
q_{\alpha}^{R} & =(c-d)(g-h) \alpha^{2}+(d g+c h-2 d h) \alpha+d h
\end{aligned}
$$

substituting $q_{\alpha}^{R}=z$, we get

$$
\alpha=\frac{-(d g+c h-2 d h)-\sqrt{(d g-c h)^{2}+4(c-d)(g-h) z}}{2(c-d)(g-h)}, \quad(\text { Omit } \alpha>1) .
$$

Therefore, the membership function of $\widetilde{Q}=\widetilde{A} \times \widetilde{B}$ is

$$
\mu_{\widetilde{Q}}(z)=\left\{\begin{array}{cc}
\frac{-(a f+b e-2 a e)+\sqrt{(a f-b e)^{2}+4(b-a)(f-e) z}}{2(b-a)(f-e)} & , a e \leq z \leq b f \\
1 & , b f \leq z \leq c g \\
\frac{-(d g+c h-2 d h)-\sqrt{(d g-c h)^{2}+4(c-d)(g-h) z}}{2(c-d)(g-h)} & , c g \leq z \leq d h \\
0 & , \text { otherwise }
\end{array}\right.
$$

Note that the core $[b f, c g]$ of $\widetilde{Q}$ is obtained by using interval methods. So, we can formulate the following result.

Proposition 1. Let $\widetilde{A}=(a, b, c, d), \widetilde{B}=(e, f, g, h)$ be two positive trapezoidal fuzzy numbers, then the membership function of their canonical product $\widetilde{Q}=\widetilde{A} \times \widetilde{B}$ is given by

\section{Example 3.1.}

$$
\mu_{\widetilde{Q}}(z)=\left\{\begin{array}{cc}
\frac{-(a f+b e-2 a e)+\sqrt{(a f-b e)^{2}+4(b-a)(f-e) z}}{2(b-a)(f-e)} & , a e \leq z \leq b f \\
1 & , b f \leq z \leq c g \\
\frac{-(d g+c h-2 d h)-\sqrt{(d g-c h)^{2}+4(c-d)(g-h) z}}{2(c-d)(g-h)} & , c g \leq z \leq d h \\
0 & , \text { otherwise }
\end{array}\right.
$$

Let $\widetilde{A}=(1,4,6,8), \widetilde{B}=(2,3,7,9)$ and $\widetilde{Q}=\widetilde{A} \times \widetilde{B}$, then by the analytic method we get

\subsubsection{Inner multiplication.}

$$
\mu_{\widetilde{Q}}(z)=\left\{\begin{array}{cl}
\frac{-7+\sqrt{25+12 z}}{6} & , 2 \leq z \leq 12 \\
1 & , 12 \leq z \leq 42 \\
\frac{17-\sqrt{1+4 z}}{4} & , 42 \leq z \leq 72 \\
0 & , \text { otherwise }
\end{array}\right.
$$

Let $\widetilde{A}=(a, b, c, d), \widetilde{B}=(e, f, g, h)$ be two trapezoidal fuzzy numbers and $\widetilde{Q}=$ $\widetilde{A} \otimes \widetilde{B}$. We set $u=\min \{a e, a h, d e, d h\}, v=\min \{b f, c f, c g, b g\}, w=\max \{b f, c f, c g, b g\}$ and $t=\max \{a e, a h, d e, d h\}$. Then, the membership function of $\widetilde{Q}$ is the following piecewise affine mapping

$$
\mu_{\widetilde{Q}}(z)=\left\{\begin{array}{cl}
\frac{z-u}{v-u} & , u \leq z \leq v \\
1 & , v \leq z \leq w \\
\frac{t-z}{t-w} & , w \leq z \leq t \\
0 & , \text { otherwise }
\end{array}\right.
$$


In particular, if $a \geq 0$ and $e \geq 0$, then $u=a e, v=b f, w=c g, t=d h$. Therefore

\section{Example 3.2. Inner product}

$$
\mu_{\widetilde{Q}}(z)=\left\{\begin{array}{cl}
\frac{z-a e}{b f-a e} & , a e \leq z \leq b f \\
1 & , b f \leq z \leq c g \\
\frac{d h-z}{d h-c g} & , c g \leq z \leq d h \\
0 & , \text { otherwise }
\end{array}\right.
$$

Let $\widetilde{A}=(1,4,6,8), \widetilde{B}=(2,3,7,9)$ and $\widetilde{Q}=\widetilde{A} \otimes \widetilde{B}$, then $\widetilde{Q}=(2,12,42,72)$ and

$$
\mu_{\widetilde{Q}}(z)=\left\{\begin{array}{cl}
\frac{z-2}{10} & , 2 \leq z \leq 12 \\
1 & , 12 \leq z \leq 42 \\
\frac{72-z}{30} & , 42 \leq z \leq 72 \\
0 & , \text { otherwise }
\end{array}\right.
$$

\section{Remark 3.3. Product of two triangular fuzzy numbers}

Let $\widetilde{A}=(a, b, d), \widetilde{B}=(e, f, h)$ be two triangular fuzzy numbers, which can be regarded as particular trapezoidal fuzzy numbers $\widetilde{A}=(a, b, c, d)$ and $\widetilde{B}=(e, f, g, h)$ , with $b=c$ and $f=g$. Let $\widetilde{Q}=\widetilde{A} \otimes \widetilde{B}$. We set $u=\min \{a e, a h, d e, d h\}, v=b f=w$ and $t=\max \{a e, a h, d e, d h\}$.

Then, the membership function of $\widetilde{Q}$ is the following piecewise affine mapping

$$
\mu_{\widetilde{Q}}(z)=\left\{\begin{array}{cl}
\frac{z-u}{v-u} & , u \leq z \leq v \\
\frac{t-z}{t-v} & , v \leq z \leq t \\
0 & , \text { otherwise }
\end{array}\right.
$$

In particular, if $a \geq 0$ and $e \geq 0$, then $u=a e, v=b f=w, t=d h$. Therefore

$$
\mu_{\widetilde{Q}}(z)=\left\{\begin{array}{cl}
\frac{z-a e}{b f-a e} & , a e \leq z \leq b f \\
\frac{d h-z}{d h-b f} & , \text { bf } \leq z \leq d h \\
0 & , \text { otherwise }
\end{array}\right.
$$

\section{Example 3.4. Product of triangular fuzzy numbers}

Let $\widetilde{A}=(2,3,5), \widetilde{B}=(3,5,6)$ be two triangular fuzzy numbers (see [4]), regarded as trapezoidal fuzzy numbers i.e $\widetilde{A}=(2,3,3,5), \widetilde{B}=(3,5,5,6)$ and $\widetilde{Q}=\widetilde{A} \times \widetilde{B}$, then by the analytic method above we get the same result as in [4]:

$$
\mu_{\widetilde{Q}}(z)=\left\{\begin{array}{cc}
\frac{-7+\sqrt{1+8 z}}{4} & , 6 \leq z \leq 15 \\
\frac{17-\sqrt{49+8 z}}{4} & , 15 \leq z \leq 30 \\
0 & , \text { otherwise }
\end{array}\right.
$$

Please notice that contrarily of the membership function of a triangular fuzzy number, this mapping is not piecewise affine. So, the canonical product of two triangular fuzzy numbers is not a triangular fuzzy number. And the canonical (or usual) multiplication does not preserve the shape of the operands (see [3]).

In the other hand, the membership function of the inner product $\widetilde{Q}=\widetilde{3} \otimes \widetilde{5}$ is given by the following

$$
\mu_{\widetilde{Q}}(z)=\left\{\begin{array}{cc}
\frac{z-6}{9} & 6 \leq z \leq 15 \\
\frac{30-z}{15} & 15 \leq z \leq 30 \\
0 & \text { otherwise }
\end{array},\right.
$$

which is piecewise affine. So the inner product of two triangular fuzzy numbers is a triangular fuzzy number. And the inner multiplication preserves the shape of the operands (see [3]). 


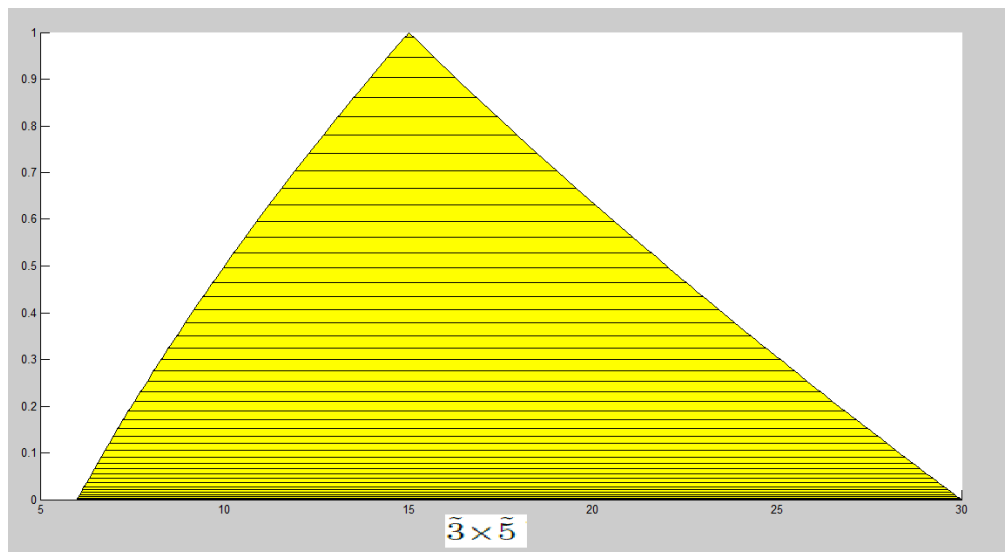

FiguRE 1. Membership function of canonical product $\tilde{3} \times \tilde{5}$ by analytical method

Now let us recall the invalid results in [4]:

Let $\widetilde{M}=\left(a_{1}, b_{1}, c_{1}\right), \widetilde{N}=\left(a_{2}, b_{2}, c_{2}\right)$ be two triangular fuzzy numbers and $\widetilde{Q}=\widetilde{M} \times$ $\widetilde{N}$. We have $\widetilde{M}_{\alpha}=\left[m_{\alpha}^{L}, m_{\alpha}^{R}\right]=\left[a_{1}+\alpha\left(b_{1}-a_{1}\right), c_{1}+\alpha\left(b_{1}-c_{1}\right)\right]$ and $\widetilde{N}_{\alpha}=\left[n_{\alpha}^{L}, n_{\alpha}^{R}\right]=$ $\left[a_{2}+\alpha\left(b_{2}-a_{2}\right), c_{2}+\alpha\left(b_{2}-c_{2}\right)\right]$. Then, $\widetilde{Q}_{\alpha}=\left[m_{\alpha}^{L}, m_{\alpha}^{R}\right] \times\left[n_{\alpha}^{L}, n_{\alpha}^{R}\right]=\left[q_{\alpha}^{L}, q_{\alpha}^{R}\right]$, where $q_{\alpha}^{L}=\min \left\{m_{\alpha}^{L} n_{\alpha}^{L}, m_{\alpha}^{L} n_{\alpha}^{R}, m_{\alpha}^{R} n_{\alpha}^{L}, m_{\alpha}^{R} n_{\alpha}^{R}\right\}$ and $q_{\alpha}^{R}=\max \left\{m_{\alpha}^{L} n_{\alpha}^{L}, m_{\alpha}^{L} n_{\alpha}^{R}, m_{\alpha}^{R} n_{\alpha}^{L}, m_{\alpha}^{R} n_{\alpha}^{R}\right\}$. The error appear clearly when the authors in [4], suppose that $c_{1} \geq b_{1} \geq a_{1}$ and $c_{2} \geq b_{2} \geq a_{2}$, and conclude that $q_{\alpha}^{L}=a_{\alpha}^{L} b_{\alpha}^{L}$ and $q_{\alpha}^{R}=a_{\alpha}^{R} b_{\alpha}^{R}$.

Their assertion is not correct, as can be shown by the following counterexample:

Example 3.5. Let $\widetilde{M}=(-6,-4,-3), \widetilde{N}=(-2,-1,2)$, then

$$
m_{\alpha}^{L}=2 \alpha-6<0, m_{\alpha}^{R}=-\alpha-3<0, n_{\alpha}^{L}=\alpha-2<0, n_{\alpha}^{R}=-3 \alpha+2,
$$

with $n_{\alpha}^{R}>0$ for all $\alpha \in\left[0, \frac{3}{2}\right]$. Then, for all $\alpha \in\left[0, \frac{3}{2}\right]$ we have

$$
m_{\alpha}^{L} n_{\alpha}^{L}>0, m_{\alpha}^{L} n_{\alpha}^{R}<0, m_{\alpha}^{R} n_{\alpha}^{L}>0, m_{\alpha}^{R} n_{\alpha}^{R}<0 .
$$

So, for all $\alpha \in\left[0, \frac{3}{2}\right]$ we have

$$
\begin{aligned}
q_{\alpha}^{L} & =\min \left\{m_{\alpha}^{L} n_{\alpha}^{L}, m_{\alpha}^{L} n_{\alpha}^{R}, m_{\alpha}^{R} n_{\alpha}^{L}, m_{\alpha}^{R} n_{\alpha}^{R}\right\} \\
& =\min \left\{m_{\alpha}^{L} n_{\alpha}^{R}, m_{\alpha}^{R} n_{\alpha}^{R}\right\} \\
& =m_{\alpha}^{L} n_{\alpha}^{R} \neq m_{\alpha}^{L} n_{\alpha}^{L},
\end{aligned}
$$

and

$$
\begin{aligned}
q_{\alpha}^{R} & =\max \left\{m_{\alpha}^{L} n_{\alpha}^{L}, m_{\alpha}^{L} n_{\alpha}^{R}, m_{\alpha}^{R} n_{\alpha}^{L}, m_{\alpha}^{R} n_{\alpha}^{R}\right\} \\
& =\max \left\{m_{\alpha}^{L} n_{\alpha}^{L}, m_{\alpha}^{L} n_{\alpha}^{R}, m_{\alpha}^{R} n_{\alpha}^{L}\right\} \neq m_{\alpha}^{R} n_{\alpha}^{R},
\end{aligned}
$$

Precisely, for $\alpha=0$, we obtain

$q_{0}^{L}=\min \{12,-12,6,-6\}=-12 ; q_{0}^{R}=\max \{12,-12,6,-6\}=12 ; m_{0}^{L} n_{0}^{L}=12 ; m_{0}^{R} n_{0}^{R}=-6$.

Then it is obvious that $q_{0}^{L} \neq m_{0}^{L} n_{0}^{L}$ and $q_{0}^{R} \neq m_{0}^{R} n_{0}^{R}$. 
The authors must suppose that the triangular fuzzy numbers $\widetilde{M}$ and $\widetilde{N}$ are positive that is $c_{1} \geq b_{1} \geq a_{1} \geq 0$ and $c_{2} \geq b_{2} \geq a_{2} \geq 0$. In this case $m_{\alpha}^{L} \geq 0, m_{\alpha}^{R} \geq$ $0, n_{\alpha}^{L} \geq 0, n_{\alpha}^{R} \geq 0$, then

$$
q_{\alpha}^{R}=m_{\alpha}^{R} n_{\alpha}^{R} \quad \text { and } \quad q_{\alpha}^{L}=m_{\alpha}^{L} n_{\alpha}^{L}
$$

3.2. Computer Drawing Method. The aim of this method is to calculate the product of two trapezoidal (respectively triangular) fuzzy numbers $\widetilde{A}, \widetilde{B}$, it is based on the calculus of $\alpha$-cuts.

Here we develop and generalize the method used in [4] for solving multiplication of two triangular fuzzy numbers.

\subsubsection{Canonical multiplication.}

Let $\widetilde{A}=(a, b, c, d), \widetilde{B}=(e, f, g, h)$ be two trapezoidal fuzzy numbers and $\widetilde{Q}=\widetilde{A} \otimes \widetilde{B}$. The method based on using the formula $\widetilde{Q}_{\alpha}=\widetilde{A}_{\alpha} \times \widetilde{B}_{\alpha}$, is as follows:

Step 1: Set $\alpha=0.01$;

Step 2: If $\alpha>1$, stop. The grey sector is the membership function of $\widetilde{Q}$. Otherwise, go to step 3 ;

Step 3: Calculate $\alpha$-cuts $\left[a_{\alpha}^{L}, a_{\alpha}^{R}\right]$ and $\left[b_{\alpha}^{L}, b_{\alpha}^{R}\right]$ of $\widetilde{A}$ and $\widetilde{B}$ respectively;

Step 4: Calculate $q_{\alpha}^{L}=\min \left\{a_{\alpha}^{L} b_{\alpha}^{L}, a_{\alpha}^{L} b_{\alpha}^{R}, a_{\alpha}^{R} b_{\alpha}^{L}, a_{\alpha}^{R} b_{\alpha}^{R}\right\}$ and $q_{\alpha}^{R}=\max \left\{a_{\alpha}^{L} b_{\alpha}^{L}, a_{\alpha}^{L} b_{\alpha}^{R}, a_{\alpha}^{R} b_{\alpha}^{L}, a_{\alpha}^{R} b_{\alpha}^{R}\right\}$;

Step 5: Set $\widetilde{Q}$ rows axis and set membership function of $\widetilde{Q}$ column axis, then create a line from $\left(q_{\alpha}^{L}, \alpha\right)$ to $\left(q_{\alpha}^{R}, \alpha\right)$

Step 6: $\alpha \leftarrow \alpha+0.01$, then go to step 2 .

\section{Example 3.6.}

Let $\widetilde{A}=(1,2,5,6), \widetilde{B}=(3,5,6,8)$, then the membership function of $\widetilde{Q}=\widetilde{A} \times \widetilde{B}$ is given in figure 2 .

\subsubsection{Inner multiplication.}

Let $\widetilde{A}=(a, b, c, d), \widetilde{B}=(e, f, g, h)$ be two trapezoidal fuzzy numbers and $\widetilde{Q}=\widetilde{A} \otimes \widetilde{B}$.

The method based on the calculus above is as follows:

Step 1: Set $\alpha=0.01$;

Step 2: If $\alpha>1$, stop. The grey sector is the membership function of $\widetilde{Q}$. Otherwise, go to step 3 ;

Step 3: Calculate $u=\min \{a e, a h, d e, d h\}, v=\min \{b f, c f, c g, b g\}, w=\max \{b f, c f, c g, b g\}$ and $t=\max \{a e, a h, d e, d h\}$

Step 4: Calculate $q_{\alpha}^{L}=u+\alpha(v-u)$ and $q_{\alpha}^{R}=t+\alpha(w-t)$;

Step 5: Set $\widetilde{Q}$ rows axis and set membership function of $\widetilde{Q}$ column axis, then create a line from $\left(q_{\alpha}^{L}, \alpha\right)$ to $\left(q_{\alpha}^{R}, \alpha\right)$;

Step 6: $\alpha \leftarrow \alpha+0.01$, then go to step 2 .

\section{Example 3.7.}

Let $\widetilde{A}=(1,2,5,6), \widetilde{B}=(3,5,6,8)$ and $\widetilde{Q}=\widetilde{A} \otimes \widetilde{B}$, then $\widetilde{Q}=(3,10,25,48)$ and

$$
\mu_{\widetilde{Q}}(z)=\left\{\begin{array}{cl}
\frac{z-3}{7} & , 3 \leq z \leq 10 \\
1 & , 10 \leq z \leq 25 \\
\frac{48-z}{23} & , 25 \leq z \leq 48 \\
0 & , \text { otherwise }
\end{array}\right.
$$

Remark 3.8. To get the correct method in the case of triangular fuzzy numbers, it is sufficient to take $b=c$ and $f=g$.

Letting $\widetilde{M}=\left(a_{1}, b_{1}, c_{1}\right), \widetilde{N}=\left(a_{2}, b_{2}, c_{2}\right)$ be two triangular fuzzy numbers (we 


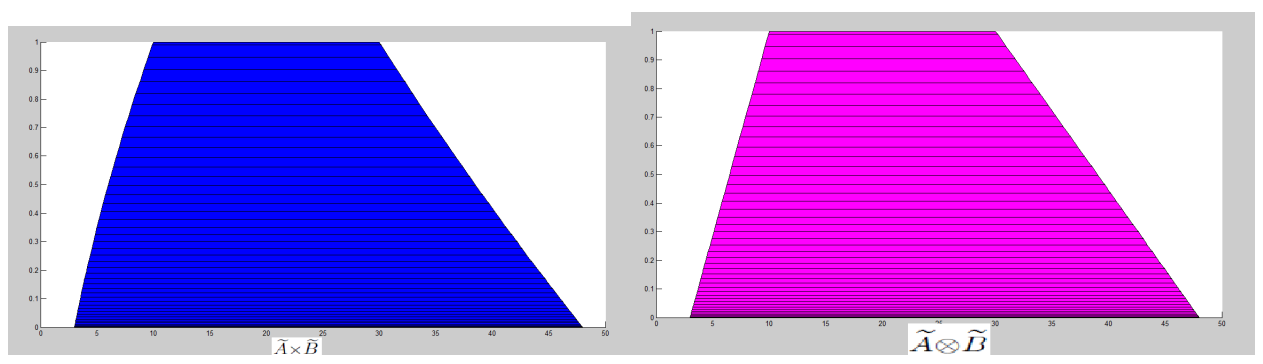

Figure 2. Canonical and inner product $\widetilde{A} \times \widetilde{B}, \widetilde{A} \otimes \widetilde{B}$ by computer drawing method

adopt the same notations in [4]). The computer drawing algorithm to calculate $\widetilde{Q}=\widetilde{M} \otimes \widetilde{N}$ is as follows:

Step 1: Set $\alpha=0.01$;

Step 2: If $\alpha>1$, stop. The grey sector is the membership function of $\widetilde{Q}$. Otherwise, go to step 3 ;

Step 3: Calculate $u=\min \left\{a_{1} b_{1}, a_{1} b_{3}, a_{3} b_{1}, a_{3} b_{3}\right\}, v=a_{2} b_{2}$ and $w=\max \left\{a_{1} b_{1}, a_{1} b_{3}, a_{3} b_{1}, a_{3} b_{3}\right\}$;

Step 4: Calculate $q_{\alpha}^{L}=u+\alpha(v-u)$ and $q_{\alpha}^{R}=w+\alpha(v-w)$;

Step 5: Set $\widetilde{Q}$ rows axis and set membership function of $\widetilde{Q}$ column axis, then create a line from $\left(q_{\alpha}^{L}, \alpha\right)$ to $\left(q_{\alpha}^{R}, \alpha\right)$

Step 6: $\alpha \leftarrow \alpha+0.01$, then go to step 2 .

\section{Example 3.9.}

Let $\widetilde{-1}=(-3,-1,2)=(-3,-1,-1,2), \widetilde{1}=(-2,1,4)=(-2,1,1,4)$ and $\widetilde{Q}=$ $\widetilde{-1} \otimes \widetilde{1}$, then $\widetilde{Q}=(-12,-1,0,8)$ and

$$
\mu_{\widetilde{Q}}(z)=\left\{\begin{array}{cc}
\frac{17-\sqrt{1-24 z}}{12} & ,-12 \leq z \leq-1 \\
\frac{2+\sqrt{-z}}{3} & ,-1 \leq z \leq 0 \\
\frac{3-\sqrt{1+z}}{3} & , 0 \leq z \leq 8 \\
0 & , \text { otherwise }
\end{array}\right.
$$
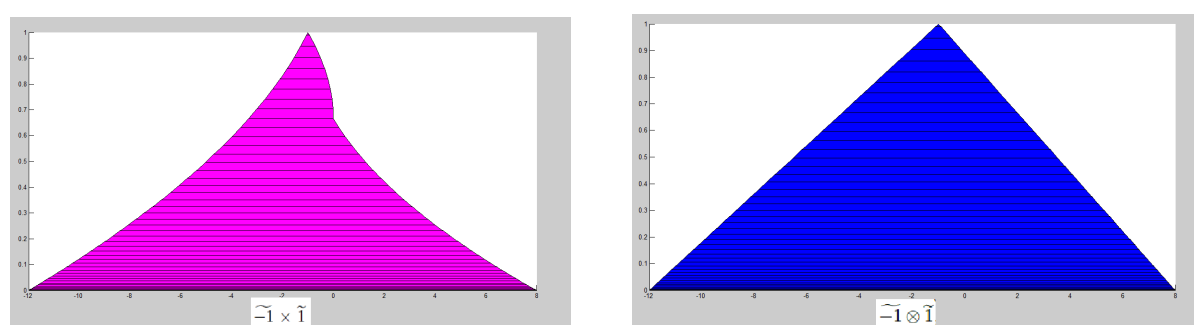

Figure 3. Canonical and inner products $\widetilde{-1} \times \widetilde{1}, \widetilde{-1} \otimes \widetilde{1}$ by computer drawing method

This figure is most precise and smooth than the following figure obtained by the same method in [4]:

Here we draw booth of the membership functions for canonical and inner product in the same figure, obtained by computer drawing method: 


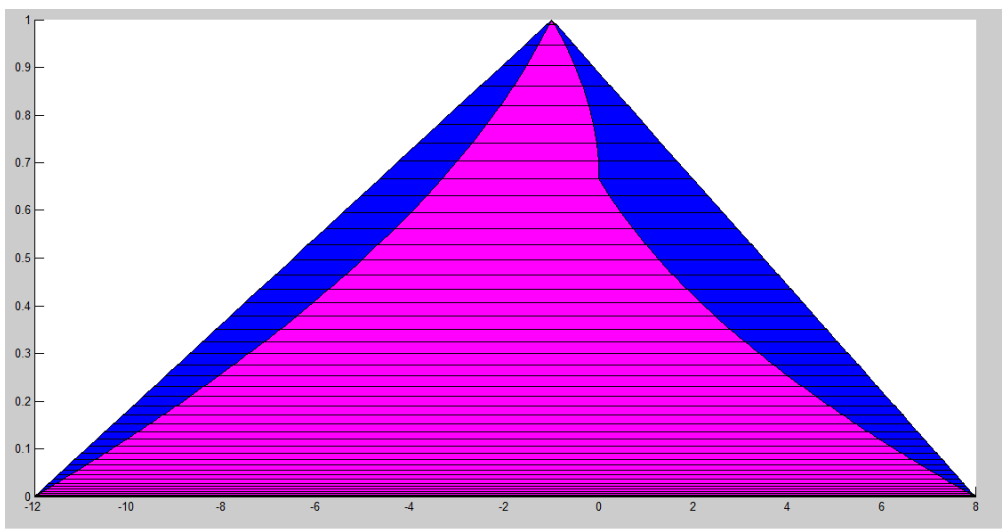

Figure 4. Membership functions of $\widetilde{-1} \times \widetilde{1}$ and $\widetilde{-1} \otimes \widetilde{1}$ by computer drawing method

3.3. Computer Simulation Method. The computer simulation method needn't calculate $\alpha$-cuts interval, it is as follows:

Step 1: Set $i=1$ and simulation times $N$;

Step 2: If $i>N$, stop. The grey sector is the membership function of $\widetilde{Q}$.Otherwise, go to step 3 ;

Step 3: generate two random numbers $x_{i}$ on the interval $[a, d]$ and $y_{i}$ on the interval $[e, h]$. Calculate $z_{i}=x_{i} y_{i}$ and $\nu_{i}=\min \left\{\mu_{\widetilde{A}}\left(x_{i}\right), \mu_{\widetilde{B}}\left(y_{i}\right)\right\}$

Step 4: Create a line from $\left(z_{i}, 0\right)$ to $\left(z_{i}, \nu_{i}\right)$, then go to step 2.

\section{Example 3.10.}

Let $\widetilde{A}=(1,2,5,6), \widetilde{B}=(3,5,6,8)$ and $\widetilde{Q}=\widetilde{A} \times \widetilde{B}$, then $\widetilde{Q}=(3,10,25,48)$ and

$$
\mu_{\widetilde{Q}}(z)=\left\{\begin{array}{cc}
\frac{-5+\sqrt{1+8 z}}{4} & , 3 \leq z \leq 10 \\
1 & , 10 \leq z \leq 30 \\
\frac{10-\sqrt{4+2 z}}{2} & , 30 \leq z \leq 48 \\
0 & , \text { otherwise }
\end{array}\right.
$$

\section{Example 3.11.}

Let $\widetilde{-1}=(-3,-1,2)=(-3,-1,-1,2), \widetilde{1}=(-2,1,4)=(-2,1,1,4)$ and $\widetilde{Q}=$ $\widetilde{-1} \otimes \widetilde{1}$, then $\widetilde{Q}=(-12,-1,0,8)$ and

$$
\mu_{\widetilde{Q}}(z)=\left\{\begin{array}{cc}
\frac{17-\sqrt{1-24 z}}{12} & ,-12 \leq z \leq-1 \\
\frac{2+\sqrt{-z}}{3} & ,-1 \leq z \leq 0 \\
\frac{3-\sqrt{1+z}}{3} & , 0 \leq z \leq 8 \\
0 & , \text { otherwise }
\end{array}\right.
$$

\section{Example 3.12.}

Let $\widetilde{3}=(2,3,5)=(2,3,3,5), \widetilde{5}=(3,5,6)=(3,5,5,6)$ and $\widetilde{Q}=\widetilde{-1} \otimes \widetilde{1}$, then $\widetilde{Q}=(3,15,15,30)$ and

$$
\mu_{\widetilde{Q}}(z)=\left\{\begin{array}{cc}
\frac{z-3}{12} & , 3 \leq z \leq 15 \\
\frac{30-z}{15} & , 15 \leq z \leq 30 \\
0 & , \text { otherwise }
\end{array}\right.
$$




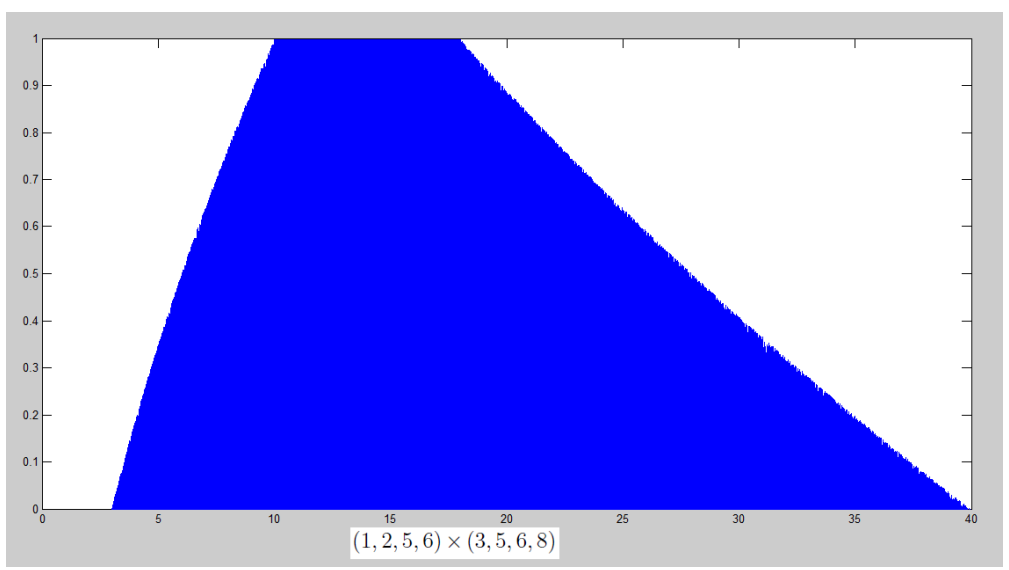

Figure 5. Membership function of $\widetilde{Q}=(1,2,5,6) \times(3,5,6,8)$ by the computer simulation method
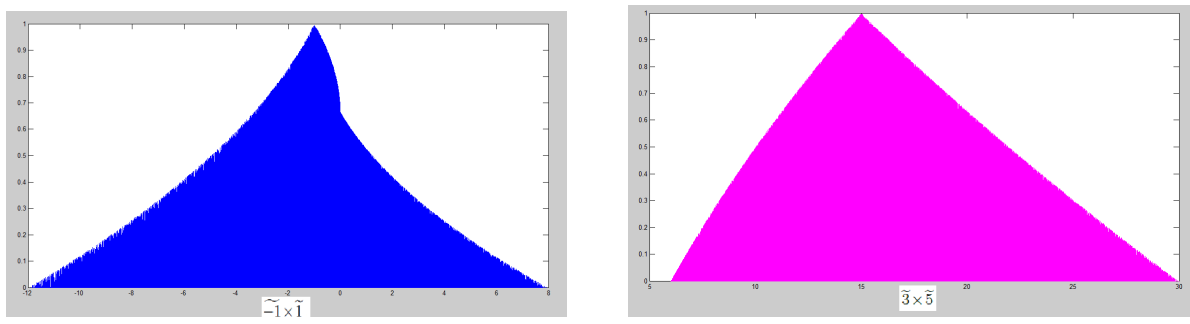

Figure 6 . Membership function of $\widetilde{-1} \times \widetilde{1}$ and $\widetilde{3} \times \widetilde{5}$ by the computer simulation method

\section{SquARE AND NTH POWER OF A TRAPEZOIDAL FUZZY NUMBER}

Let $\widetilde{A}=(a, b, c, d)$ be a trapezoidal fuzzy number, we denote respectively $\widetilde{A}^{2}=$ $\widetilde{A} \otimes \widetilde{A}$ and $\widetilde{A}^{(2)}=\widetilde{A} \otimes \widetilde{A}$ its canonical and inner square.

Proposition 2. The membership function of the canonical square $\widetilde{A}^{2}$ of a positive trapezoidal fuzzy number $\widetilde{A}=(a, b, c, d)$ is given by

$$
\mu_{\widetilde{A}^{2}}(z)=\left\{\begin{array}{cl}
\frac{-a+\sqrt{z}}{b-a} & , a^{2} \leq z \leq b^{2} \\
1 & , b^{2} \leq z \leq c^{2} \\
\frac{d-\sqrt{z}}{d-c} & , c^{2} \leq z \leq d^{2} \\
0 & , \text { otherwise }
\end{array}\right.
$$

Proof. It results immediately from the proposition 1.

Corollary 4.1. The membership function of the canonical square $\widetilde{A}^{2}$ of a positive triangular fuzzy number $\widetilde{A}=(a, b, c)$ is given by

$$
\mu_{\widetilde{A}^{2}}(z)=\left\{\begin{array}{cc}
\frac{-a+\sqrt{z}}{b-a} & , a^{2} \leq z \leq b^{2} \\
\frac{c-\sqrt{z}}{c-b} & , b^{2} \leq z \leq c^{2} \\
0 & , \text { otherwise }
\end{array}\right.
$$


Proof. It results obviously from the proposition 2 .

Proposition 3. The membership function of the inner square $\widetilde{A}^{(2)}$ of a positive trapezoidal fuzzy number $\widetilde{A}=(a, b, c, d)$ is given by

$$
\mu_{\widetilde{A}^{(2)}}(z)=\left\{\begin{array}{cl}
\frac{z-a^{2}}{b^{2}-a^{2}} & , a^{2} \leq z \leq b^{2} \\
1 & , b^{2} \leq z \leq c^{2} \\
\frac{d^{2}-z}{d^{2}-c^{2}} & , c^{2} \leq z \leq d^{2} \\
0 & , \text { otherwise }
\end{array}\right.
$$

It results immediately from the results in paragraph ??

Corollary 4.2. The membership function of the inner square $\widetilde{A}^{(2)}$ of a positive triangular fuzzy number $\widetilde{A}=(a, b, c)$ is given by

$$
\mu_{\widetilde{A}^{(2)}}(z)=\left\{\begin{array}{cl}
\frac{z-a^{2}}{b^{2}-a^{2}} & , a^{2} \leq z \leq b^{2} \\
\frac{c^{2}-z}{c^{2}-b^{2}} & , b^{2} \leq z \leq c^{2} \\
0 & , \text { otherwise }
\end{array}\right.
$$

Proof. It results obviously from the proposition 3.

\section{Example 4.3.}

Let $\widetilde{A}=(2,3,5,6)$, then its inner square $\widetilde{A}^{(2)}=(4,9,25,36)$ and the membership function of its canonical square is given by

\section{Example 4.4.}

$$
\mu_{\widetilde{A}^{2}}(z)=\left\{\begin{array}{cl}
\sqrt{z}-2 & , 4 \leq z \leq 9 \\
1 & , 9 \leq z \leq 25 \\
6-\sqrt{z} & , 25 \leq z \leq 36 \\
0 & , \text { otherwise }
\end{array}\right.
$$

Let $\widetilde{A}=(2,3,5)$, then its inner square $\widetilde{A}^{(2)}=(4,9,25)$ and the membership function of its canonical square is given by

$$
\mu_{\widetilde{A}^{2}}(z)=\left\{\begin{array}{cl}
\sqrt{z}-2 & , 4 \leq z \leq 9 \\
\frac{5-\sqrt{z}}{2} & , 9 \leq z \leq 25 \\
0 & , \text { otherwise }
\end{array}\right.
$$
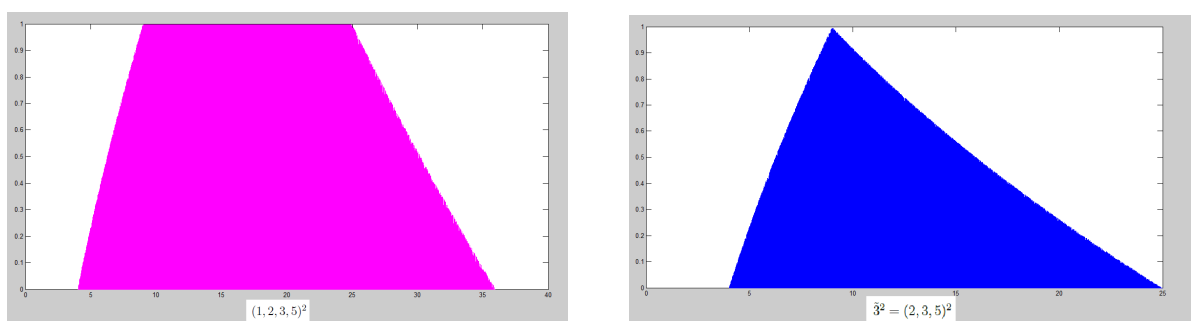

Figure 7 . Canonical squares of $(1,2,3,5)$ and $\tilde{3}=(2,3,5)$ by the computer simulation method

Let $\widetilde{A}=(a, b, c, d)$ be a trapezoidal fuzzy number and $n \geq$ be a positive integer. By recurrence, we define respectively $\widetilde{A}^{n}=\widetilde{A}^{n-1} \otimes \widetilde{A}$ and $\widetilde{A}^{(n)}=\widetilde{A}^{(n-1)} \otimes \widetilde{A}$ its canonical and inner $n$th power. 


\section{Example 4.5.}

Let $\widetilde{A}=(2,3,5,6)$, then its inner $n$th power is the trapezoidal fuzzy number $\widetilde{A}^{(n)}=$ $\left(2^{n}, 3^{n}, 5^{n}, 6^{n}\right)$. Using the analytical method, we get the membership function of its canonical $n$th power:

$$
\mu_{\widetilde{A}^{2}}(z)=\left\{\begin{array}{cl}
\sqrt[n]{z}-2 & , 2^{n} \leq z \leq 3^{n} \\
1 & , 3^{n} \leq z \leq 5^{n} \\
6-\sqrt[n]{z} & , 5^{n} \leq z \leq 6^{n} \\
0 & , \text { otherwise }
\end{array}\right.
$$

\section{Conclusion}

We have presented three numerical methods for solving inner and canonical multiplication on trapezoidal fuzzy numbers. As it is remarked in [4], the analytical method is more precise and the computer drawing method is simple, but there are both based on calculus of $\alpha$-cuts interval, which seems difficult and hard in certain cases. The computer simulation method is the most simple, but it gives a membership function rough and not very smooth. For future research, one can apply these methods to solve nonlinear fuzzy differential equations, which involves the powers of trapezoidal or triangular fuzzy numbers.

\section{REFERENCES}

[1] A. Bansal, Trapezoidal Fuzzy Numbers (a,b,c,d): Arithmetic Behavior, Inernational Journal of Physical and mathematical Sciences, 2011, pp.39-44 (2011).

[2] D. Dubois \& H. Prade, Operations on Fuzzy Numbers, International Journal of Systems Sciences, 9, pp.613-626 (1978).

[3] J. Fodor \& B. Bede, Arithmetics With Fuzzy Numbers: A Comparative Overview.

[4] S. Gao, Z. Zhang \& C. Cao, Multiplicatin Operation on Fuzzy Numbers, Journal of Software, 2009, pp.331-338 (2009).

[5] S. Heilpern, Rpresetation and Application of Fuzzy Numbers, Fuzzy sets and Systems, 91, pp.259-268 (1997).

[6] G.J. Klir, Fuzzy Arithmetic with Requisite Constraints, Fuzzy sets and Systems, 91, pp.165175 (1997).

[7] G.J. Klir, Fuzzy Sets: An Overview of Fundmentals, Applications and Personal Views, Beijing Normal University Presse, 2000, pp.44-49 (2000).

[8] H.M. Lee \& L. Lin , Using Weighted Triangular Fuzzy Numbers to Evaluate the rate of aggregative risk in software developpement, Proceeding of 7 th International Conference of Machine Learning and Cybernetics, 2008, 7, pp.3762-3767.

[9] S. Mahanta, R. Chuta \& H.K. Baruah, Fuzzy Arithmetic Without Using the Method of $\alpha$-Cuts, International Journal of Latest Trents in Computing, 1 , pp.73-80, (2010).

[10] N. Gani, \& S.N.M. Assarudeen, A New Operation on Triangular Fuzzy Number for Solving Linear Programming Problem, Applied Mathematical Sciences, pp.525-532, 2012.

[11] L.A. Zadeh, Fuzzy Set, Information and Control, 8, pp.339-353, 1965.

(E. Eljaoui) LMaCS - Sultan Moulay Slimane University, BP 523, 23000, Beni Mellal, MOROCCO

E-mail address, E. Eljaoui: eljaouihass@gmail.com

(S. Melliani) LMaCS - Sultan Moulay Slimane University, BP 523, 23000, Beni Mellal, MOROCCO

E-mail address, S. Melliani: saidmelliani@gmail.com

(L. S. Chadli) LMaCS - Sultan Moulay Slimane University, BP 523, 23000, Beni Mellal, MOROCCO

E-mail address, L. S. Chadli: sa.chadli@yahoo.fr 